\title{
Finite Element Based Optimal Design Approach For High Voltage Pulse Transformers
}

\author{
D. Aguglia \\ CERN, Geneva, Switzerland \\ S. Candolfi, P. Viarouge, J. Cros \\ LEEPCI Lab., Quebec, Canada
}

Keywords: CLIC

\begin{abstract}
This paper presents an optimal design methodology of monolithic high voltage pulse transformers based on the direct 2D FEA identification of the electrical equivalent circuit parameters. This method is applied to the preliminary optimal design of the monolithic high voltage pulse transformer for the future CLIC modulators under study at CERN. The feasibility of such a transformer with tight specifications is demonstrated. The predicted performances obtained with the direct 2D FEA optimization process is validated by $3 \mathrm{D}$ FEA simulation.
\end{abstract}

Presented at: IEEE pulsed power and plasma science conference (PPPS),

San Francisco, USA, June 2013

Geneva, Switzerland

November, 2014 


\title{
Finite Element Based Optimal Design Approach For High Voltage Pulse Transformers
}

\author{
Sylvain Candolfi ${ }^{1}$, Member, IEEE, Philippe Viarouge ${ }^{1}$, Member, IEEE, \\ Davide Aguglia ${ }^{2}$, Member, IEEE, Jérôme $\mathrm{Cros}^{1}$, Member, IEEE \\ ${ }^{1}$ LEEPCI Lab., Electrical and Computer Eng. Dept., Laval University, G1V 0A6 Quebec (QC), Canada \\ Emails: sylvain.candolfi.1@ulaval.ca, philippe.viarouge@gel.ulaval.ca, jerome.cros@gel.ulaval.ca \\ ${ }^{2}$ CERN - European Organization for Nuclear Research, Technology Dept., Electric Power Converter Group \\ CH-1211 Geneva 23, Switzerland Email: davide.aguglia@cern.ch
}

\begin{abstract}
This paper presents an optimal design methodology of monolithic high voltage pulse transformers based on the direct 2D FEA identification of the electrical equivalent circuit parameters. This method is applied to the preliminary optimal design of the monolithic high voltage pulse transformer for the future CLIC modulators under study at CERN. The feasibility of such a transformer with tight specifications is demonstrated. The predicted performances obtained with the direct 2D FEA optimization process is validated by 3D FEA simulation.
\end{abstract}

\section{INTRODUCTION}

High voltage pulse transformers are widely used in klystron modulators. For these topologies, the quality of the modulator output voltage is strongly dependent on the pulse transformer performance. An optimal design process is a necessity, particularly when the specifications of the output voltage are tight such as in the case of the CLIC klystron modulator under study at CERN [1]. With these new specifications far above the present state of the art, the feasibility of the monolithic pulse transformer based modulator topology needs the development of a specific and accurate design methodology of High Power \& High Voltage Pulse Transformers. This paper presents the development of a CAD environment that is using a dimensioning model based on 2D Finite Element Analysis (FEA) only and a non-linear optimization procedure. For each iteration of the non-linear optimization process, the transformer parameters used to compute the objective and constraints functions are directly derived from the 2D FEA dimensioning model.

\section{High VOLTAGE PULSE TRANSFORMERS IN KLYSTRON MODULATORS}

\section{A. Modulator topology using monolithic pulse transformer}

Fig. 1 presents the structure of a solid state klystron modulator using a monolithic pulse transformer based topology. It is composed of a capacitive storage charger and a pulse forming system. The main capacitor bank is charged at $10 \mathrm{kV}$ by the capacitor charger power converter.

A high voltage fully controllable solid state switch (made of IGBT or IGCT stacks) is used to apply the capacitor voltage

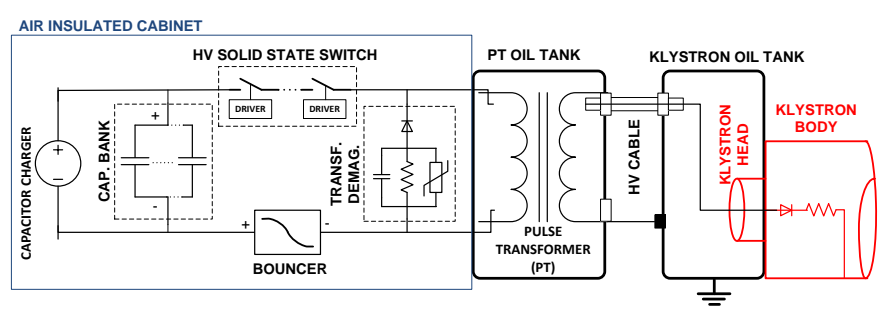

Fig. 1. Monolithic pulse transformer based topology

to a pulse transformer that rises up the voltage to get a $150 \mathrm{kV}$ output voltage pulse. At the end of the pulse, the main switch is opened and the core magnetizing energy is absorbed by an undershoot network. The bouncer is used to compensate the discharge droop of the main capacitor bank during the pulse. It can be passive (realized with reactive components) or active (realized with an auxiliary power converter) [2] [1]. A fast solid state crowbar is also placed in parallel with the capacitor bank to provide emergency fast discharge capability.

\section{B. High Voltage Pulse Transformer Structure}

The HV (High Voltage) pulse transformer is immersed in an oil tank. Its core is made of high grade laminated material. Several winding configurations can be adopted [4], but the conventional topology is presented in Fig. 2. Two primary windings $P_{1}$ and $P_{2}$ are placed on each core leg and connected in parallel. With the usual transformer specifications, each section can be realized with only one single layer of conductors. The secondary HV winding is composed of two sections SHV and SLV wound above the primary windings on each core leg and connected in series.

Both secondary sections are using a disc winding configuration with several horizontal discs spaced along the axial length of the core leg. In each disc, turns are wound in a radial direction, with one turn per layer. With such a configuration, additional insulation between layers is minimized. The use of a single layer winding on the primary side and a disc winding on the secondary side usually leads to a minimization 


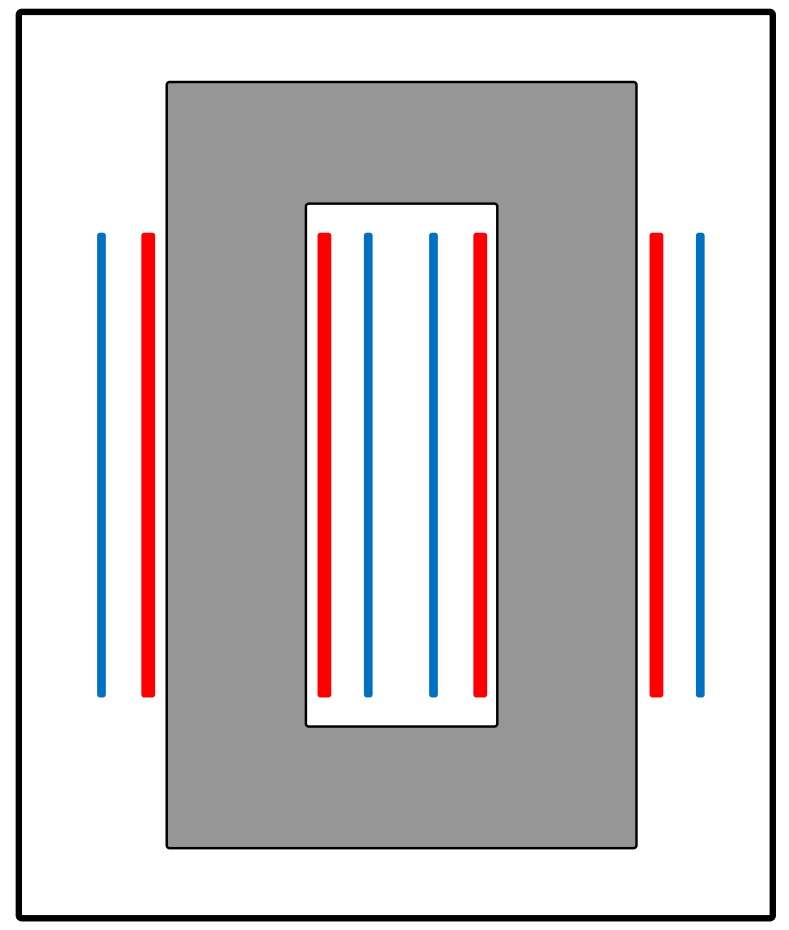

Fig. 2. Simplified Structure of Oil Immersed High Voltage Pulse Transformer in its Tank (Primary Windings in blue, Secondary windings in red)

of the inter-turn winding capacitance. Along each core leg, the space between the primary and secondary coils can be constant or adapted to try to operate the transformer with a constant voltage gradient between them by use of conical shape of the secondary windings in order to minimize the leakage inductance [5]. The core is also equipped with an auxiliary winding supplied by a DC current source associated to an external inductor to provide a pre-magnetization of the core with a negative flux density before application of the voltage pulse to the primary windings. With such an arrangement, a bipolar magnetic flux excursion $\Delta B=2 B_{s}$ (where $B_{s}$ is the saturation flux density of the core magnetic material) is obtained when the unipolar voltage pulse is applied to the primary windings and the pulse transformer core mass and volume can be minimized [4].

The grounding connections of the transformer core, tank and windings are presented on Fig. 3.

\section{HV Pulse Transformer Equivalent Circuit}

Because the modulator monolithic transformer is supplied with a high voltage and operated in pulsed mode, the distributed capacitances between the primary and secondary windings, between the windings and the grounded core and tank cannot be neglected. They have a high influence on the dynamic characteristics of the output voltage pulse, according to the pulse length and the modulator instantaneous pulse power. In the pulse length range imposed by the applications

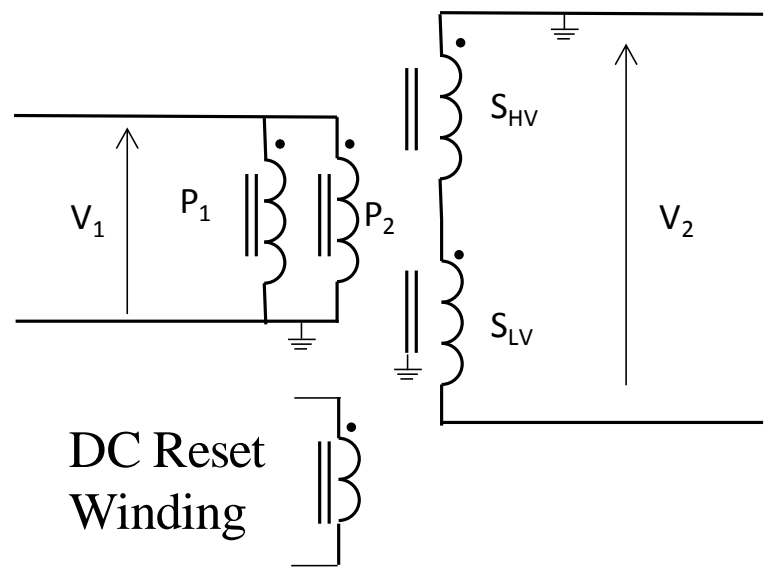

Fig. 3. High Voltage Pulse Transformer Winding Connections

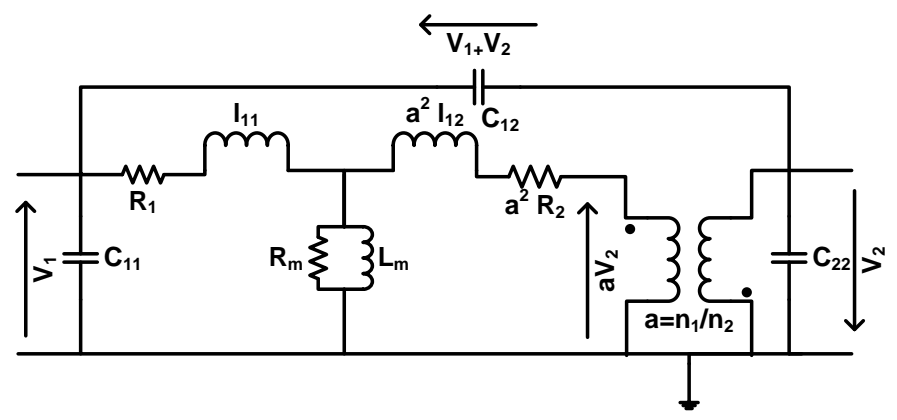

Fig. 4. Electrical Equivalent Circuit of High Voltage Pulse Transformer

under study, the lumped-parameter equivalent circuit of Fig. 4 can be adopted to model a transformer with distributed capacitance. The distribution of the magnetic energy stored in the transformer is represented by a magnetizing inductance and two leakage inductances.

With such an assumption, the propagation phenomenon is neglected and the electromagnetic model can be decoupled in an electrostatic model and a magnetostatic model [5] [6]. If a linear-distributed voltage in windings is assumed, it is possible to establish a dimensioning model of the different components of the electrical equivalent circuit (see Fig. 5). Transformer capacitances and inductances can be respectively derived from the computation of the electric and magnetic energy stored in different regions of the device [5].

For practical presentation purpose, the following figures present the electrical equivalent circuit of a non-inverting transformer only; the case of an inverting transformer is similar in terms of circuit structure.

\section{High Voltage Pulse Transformer DiMENSIONING MODEL}

\section{A. HV Pulse Transformer Design Problem}

In the modulator topology under study, the performance requirements of the high voltage monolithic pulse transformer 


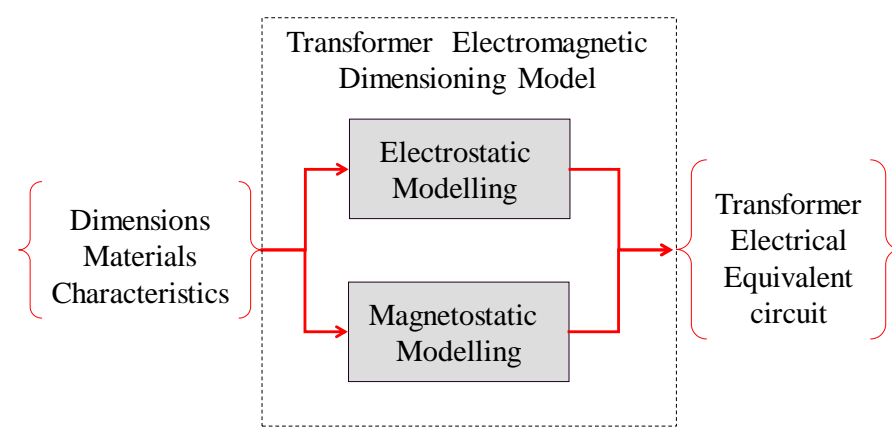

Fig. 5. HV Pulse Transformer Electromagnetic Dimensioning Model derived from the modeling assumptions

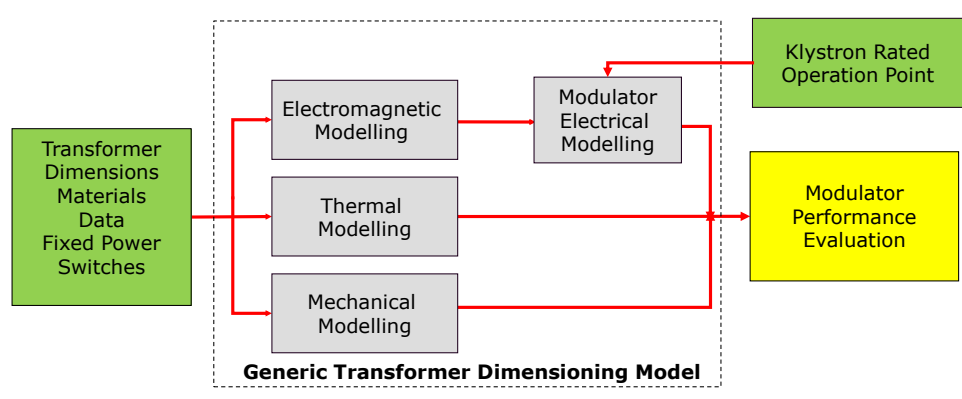

Fig. 6. High Voltage Pulse Transformer Generic Dimensioning Model

are an important issue in terms of dynamical specs, droop, efficiency, power to volume and power to mass ratios, long term reliability and cost [1]. With the required pulse width and repetition rate, the minimization of the total power consumption highly depends on the rise and fall times of the output voltage pulse. The output voltage pulse quality is strongly dependent on pulse transformer leakage inductances \& parasitic capacitances that must be controlled during the design process. An accurate dimensioning model and an optimal design process of such transformers are required.

\section{B. HV Pulse Transformer Generic Dimensioning Model}

The transformer dimensioning model can be derived from the electrical equivalent circuit and the determination of its components in terms of dimensions and material data. A generic dimensioning model of the monolithic HV pulse transformer is presented on Fig. 6. It includes the preceding electromagnetic dimensioning model, a simplified thermal modeling tool and a simple mechanical modeling tool used to evaluate the structural behavior of the transformer operated in pulsed mode. The output voltage pulse is determined by simulation of the modulator electrical modeling that is including a transformer equivalent circuit derived from the electromagnetic dimensioning model.

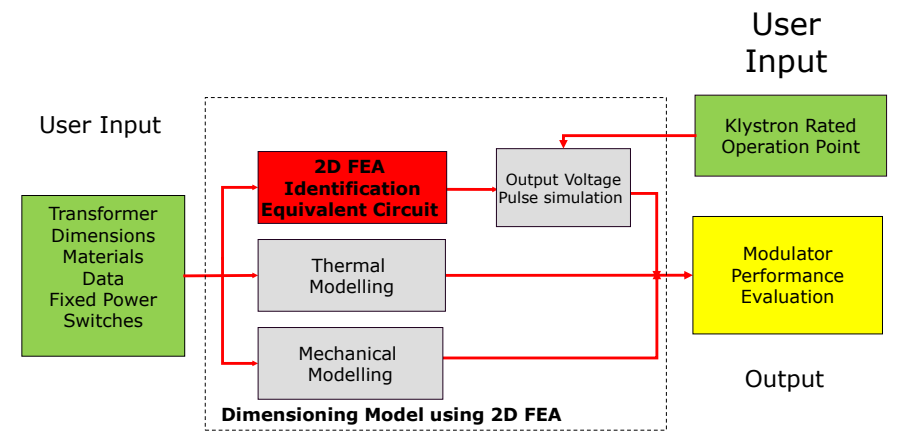

Fig. 7. High Voltage Pulse Transformer Dimensioning Model using 2D FEA

\section{Optimal Design of High Voltage Pulse TRANSFORMER USING 2D FINITE ElEMENT ANALYSIS}

In the case of high voltage transformers, it is difficult to obtain an analytical electromagnetic dimensioning model that is sufficiently accurate to perform an optimal design of the transformer in terms of dynamical performances. In a previous design approach developed by the authors, field computation techniques based on 2D FEA have been already implemented in the CAD environment to validate or correct the analytical electromagnetic dimensioning model [5]. In this paper a new efficient optimal design methodology is presented that is using a direct 2D FEA electromagnetic dimensioning model during the transformer optimization process. The dimensioning model based on 2D FEA only is presented on Fig. 7.

The simulated experiment concept has been applied to perform direct FEA identification tests of the electrical equivalent circuit components. With such an approach, no simplified analytical expressions of the inductances and capacitances are used.

\section{A. 2D FEA identification of transformer inductances}

Two FEA identification tests based on magnetostatic field computations are performed for the determination of the magnetizing \& leakage inductances:

- Open Secondary FEA test: the magnetizing inductance is derived from the FEA computation of the magnetic energy stored during transformer no-load operation.

- Short-circuit FEA test: the total leakage inductance is derived from the FEA computation of the magnetic energy stored during transformer short-circuit operation.

\section{B. 2D FEA identification of transformer capacitances}

2D Electrostatic field computations have been performed to identify the three lumped capacitors of the electrical equivalent circuit of Fig. 4. The three lumped capacitors $C_{1}, C_{2} \& C_{12}$ can be derived from the electrostatic FEA simulations of three different tests presented in Fig. 8: (a) Secondary short circuited \& grounded, (b) Primary short circuited \& grounded, (c) Primary \& secondary with $V_{2 n}$ linear voltage distribution. One can compute the three capacitances from the three values 


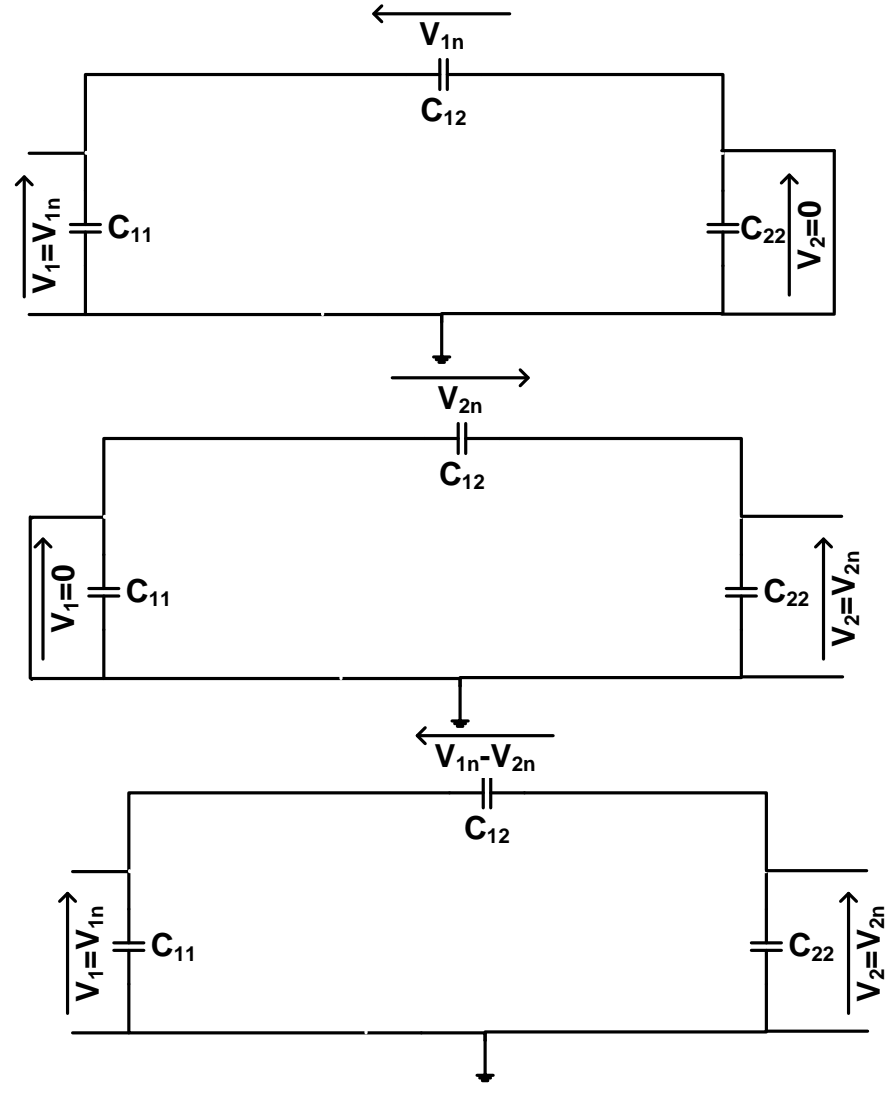

Fig. 8. Description of the operation conditions of the three FEA identification tests : (a) $V_{1}=V_{1 n} V_{2}=0 W_{2 c c}$, (b) $V_{1}=0 V_{2}=V_{2 n} W_{1 c c}$, (c) $V_{1}=V_{1 n} V_{2}=V_{2 n} W_{122}$

$W_{2 c c}, W_{1 c c} \& W_{122}$ of total electrical energy derived from the electrostatic FEA simulations.

$$
\begin{gathered}
C_{1}+C_{12}=\frac{2 W_{2 c c}}{V_{1}^{2}} \quad C_{2}+C_{12}=\frac{2 W_{1 c c}}{V_{2}^{2}} \\
V_{1}^{2} C_{1}+V_{2}^{2} C_{2}+\left(V_{2}-V_{1}\right)^{2} C_{12}=2 W_{122}
\end{gathered}
$$

The distributions of voltage in Pulse Transformer tank corresponding to the three identification tests (a) (b) \& (c) are presented in Fig. 9. When a linear vertical rated voltage distribution is imposed along the height of the primary \& secondary coils, one obtains the distribution of Fig. 9(c).

\section{High Voltage Pulse Transformer Design with Global Op- timization Approach Using 2D FEA}

Fig. 10 presents the structure of the final CAD environment with the global optimization approach using the previous 2D FEA electromagnetic dimensioning model. The identified parameters derived from 2D FEA are used to compute the objective and constraints functions of the non-linear optimization procedure to deliver the optimal transformer design. With a suitable selection of the dimensional variables and an adequate choice of the non-linear optimization method in order to get rid (a)

(b)

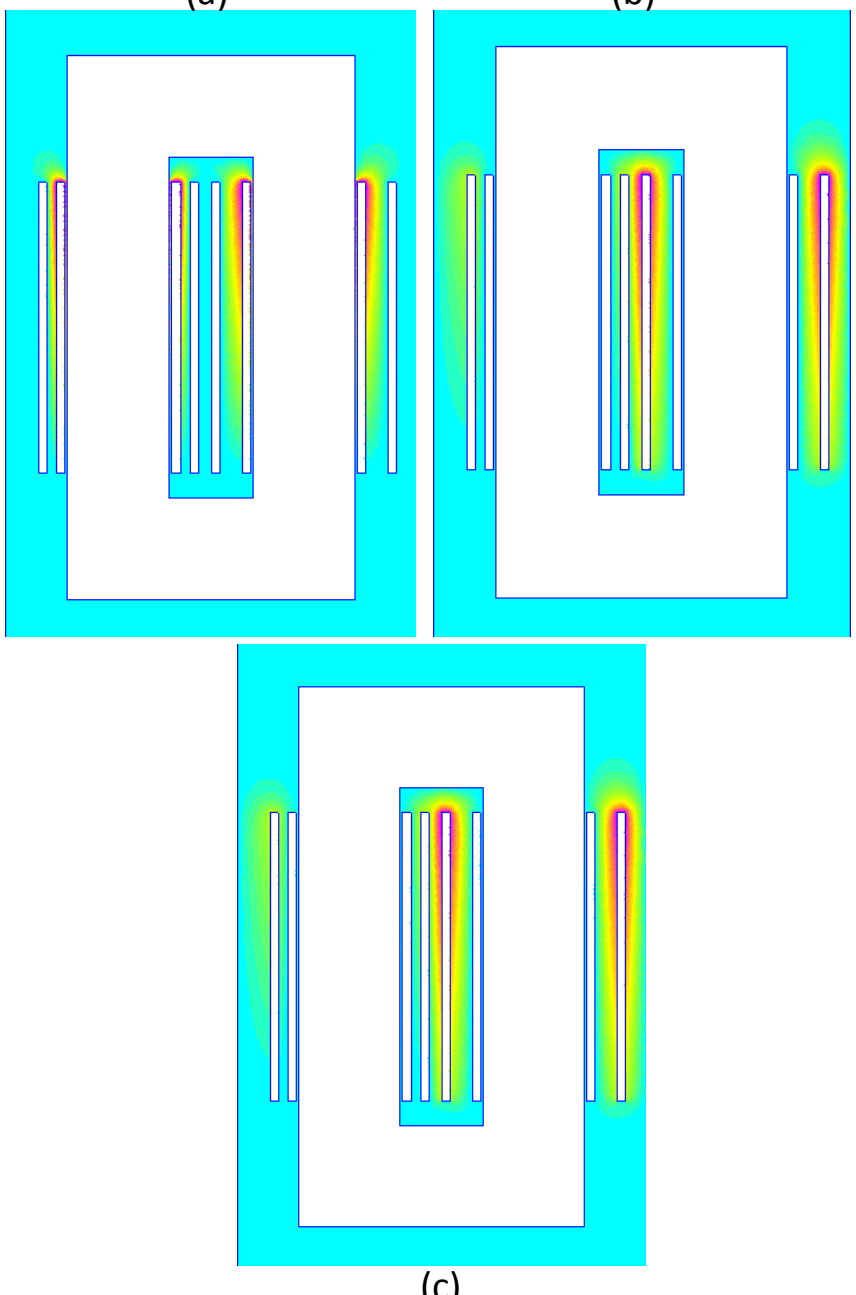

Fig. 9. Distribution of Voltage in Pulse Transformer Tank for the 3 identification tests : (a) $V_{1}=V_{1 n} V_{2}=0 W_{2 c c}$ (b) $V_{1}=0 V_{2}=V_{2 n} W_{1 c c}$ (c) $V_{1}=V_{2 n} V_{2}=V 2 n W_{122}$

of the FEA mesh discretization influence, the optimal design solution is achieved.

\section{EXAMPLE OF PULSE TRANSFORMER OPTIMIZATION}

The proposed methodology has been applied to investigate the preliminary design of the pulse transformer for the CLIC klystron modulator under study at CERN [1][3]. The Compact Linear Collider (CLIC) will need 300MW of average power feeding 1638 klystron modulators with tight dynamical specifications in terms of rise time for a relatively long flat-top width (Fig. 11) and high efficiency for minimizing the total power consumption of the accelerator complex. The modulators will be operated at $150 \mathrm{kV} / 160 \mathrm{~A}(24 \mathrm{MW})$ in pulsed mode.

\section{A. Preliminary CLIC Pulse Transformer Optimal Design}

The constraints of the optimization problem listed in Table I are the specifications of the modulator, the transformer overall 


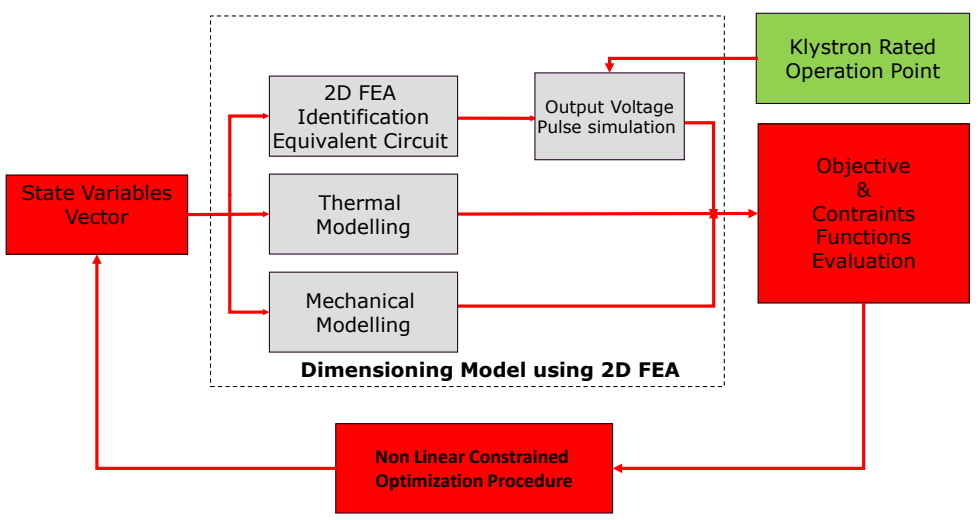

Fig. 10. High Voltage Pulse Transformer Design with Global Optimization Approach Using 2D FEA

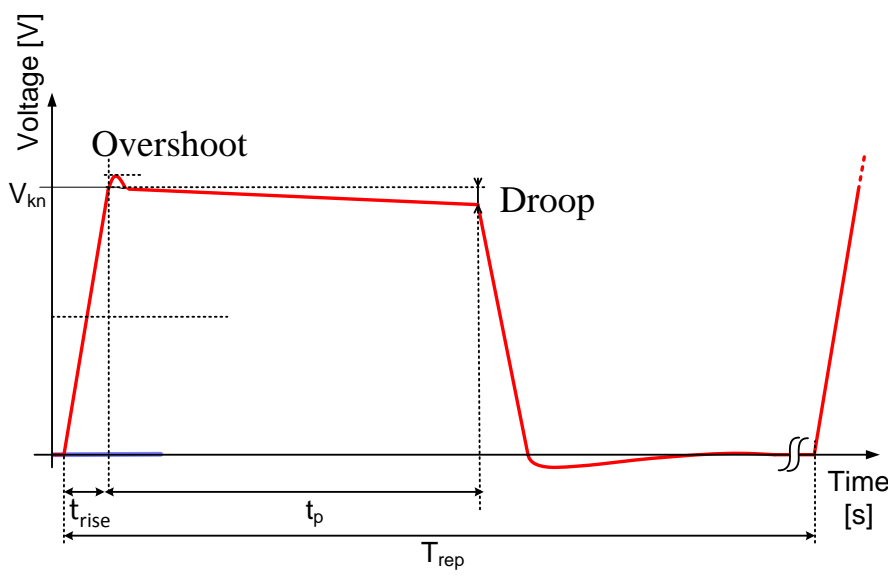

Fig. 11. Definition of pulse characteristics

dimensions and copper losses and the characteristics of the transformer magnetic and insulation materials.

The three state variables of the transformer design optimization problem are:

- The primary turn number

- The height of the coils

- The core width

The problem objective function to minimize is the total weight of the transformer active parts excluding the oil in the tank. Two transformer configurations have been optimized: one configuration with a constant space between the primary and secondary coils, one configuration with a conical shape of the secondary windings. The optimization procedure was operated in multistart mode to avoid the convergence on local minima of the objective function in the space of solutions bounded by the constraints. The feasibility of the CLIC monolithic pulse transformer with the the imposed specifications has been demonstrated. All the constraints including the voltage rise time and voltage overshoot specifications are respected for the optimal solutions found for both configurations under study. The optimal transformer weights are listed on Table III. The conical winding configuration complicates the transformer
TABLE I

SPECIFICATIONS AND CONSTRAINTS OF CLIC PULSE TRANSFORMER OPTIMAL DESIGN

\begin{tabular}{|l|c|c|}
\hline Pulse specification & Value & Unit \\
\hline Peak output power & 24 & $\mathrm{MW}$ \\
Primary nominal voltage $V_{1 n}$ & 15 & $\mathrm{kV}$ \\
Secondary nominal voltage $V_{2 n}$ & 150 & $\mathrm{kV}$ \\
Pulse length $t_{p}$ & 140 & $\mu \mathrm{s}$ \\
Voltage pulse rise time $t_{\text {rise }}$ & $<5$ & $\mu \mathrm{s}$ \\
Voltage pulse overshoot & $<5 \%$ & - \\
Pulse repetition rate $\frac{1}{T_{r e p}}$ & 50 & $\mathrm{~Hz}$ \\
\hline Material specifications & Value & $\mathrm{Unit}$ \\
\hline Breakdown field of insulating material & 9 & $\mathrm{MV} / \mathrm{m}$ \\
Relative permittivity of insulating material & 3 & - \\
Relative permeability of magnetic material & 4000 & - \\
Saturation flux density of magnetic material & 1.15 & $\mathrm{~T}$ \\
\hline Constraint & Value & $\mathrm{unit}$ \\
\hline Copper losses & $<3 \%$ & - \\
Max. overall dimensions & $1 \times 0.5 \times 1.2$ & $\mathrm{~m}$ \\
\hline
\end{tabular}

TABLE II

CHARACTERISTICS OF OPTIMAL CLIC TRANSFORMER CONFIGURATION WITH CONSTANT SPACE BETWEEN PRIMARY AND SECONDARY COILS.

\begin{tabular}{|l|c|c|c|c|}
\hline Characteristic & 2D FEA & 3D FEA & Unit & Difference [\%] \\
\hline Magnetizing inductance $L_{m}$ & 0.148 & 0.151 & $\mathrm{H}$ & $-2 \%$ \\
Leakage inductance $l_{11}+a^{2} l_{12}$ & 21.3 & 24.2 & $\mu \mathrm{H}$ & $-14 \%$ \\
Primary capacitance $C_{11}$ & 2.43 & 2.55 & $\mathrm{nF}$ & $-5 \%$ \\
Secondary capacitance $C_{22}$ & 1080 & 775 & $\mathrm{pF}$ & $28 \%$ \\
Inter-windings capacitance $C_{12}$ & 907 & 629 & $\mathrm{pF}$ & $31 \%$ \\
Voltage pulse rise time & 5 & 5.15 & $\mu \mathrm{s}$ & $3 \%$ \\
Voltage pulse overshoot & 0 & 0 & $\%$ & $0 \%$ \\
\hline
\end{tabular}

production process but it does not significatively increase the performance.

\section{B. Validation of the optimal design by $3 D$ FEA simulation}

The optimal solution of the first transformer configuration was simulated with a 3D Finite Element Analysis code to further investigate the influence of the 2D FEA assumptions used in the proposed design methodology. The results of the 2D and 3D FEA simulations in terms of equivalent circuit parameters and voltage pulse characteristics can be compared in Table II. One can notice that the difference on the pulse rise time and overshoot are 3\% and $0 \%$ only. This is an important result for the validation of the use of the $2 \mathrm{D}$ assumption in the proposed transformer optimization process because time consuming 3D FEA simulations are not well adapted to an iterative process. The error on the rise time is low despite the fact that the most important errors related to the $2 \mathrm{D}$ assumption occur on the capacitance values. The rise time depends on both capacitance and inductance values and in the the absence of overshoot imposed by the specifications constraints, the errors on the inductance and capacitance values partially compensate each other in the computation of the rise time. Both 3D and 2D models are then in accordance when no pulse overshoot is allowed.

The induction spatial distribution plot for short circuit operation (Fig. 12) and the electric field spatial distribution plot for the test $V_{1}=0$ and $V_{2}=150 \mathrm{kV}$ (Fig. 13) with 2D FEA and 3D FEA can be compared. On the plane where 2D FEA 

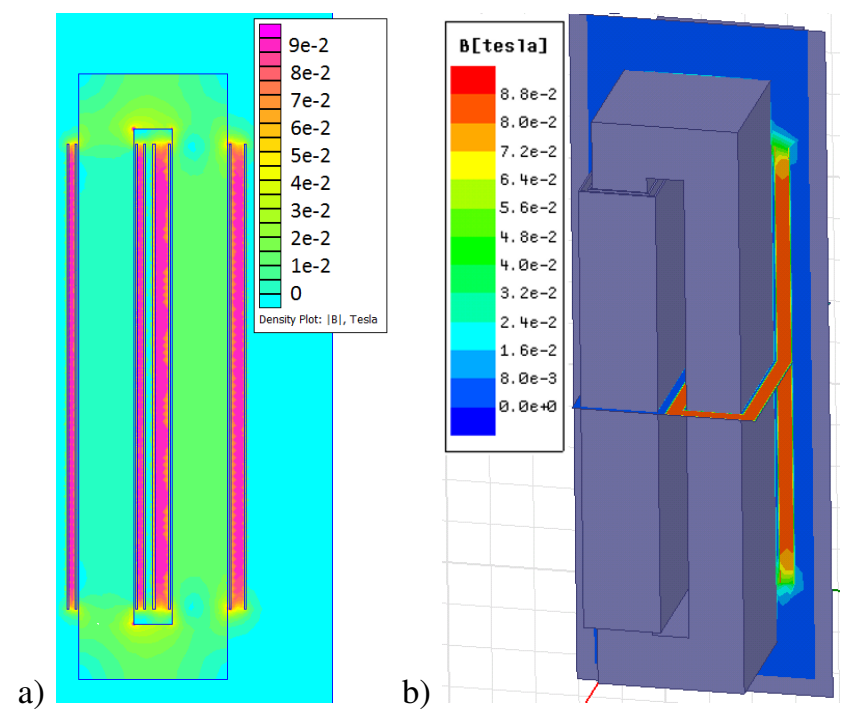

Fig. 12. Comparison between $2 \mathrm{D}$ a) and 3D b) FEA induction plots for short circuit operation. For 3D results, coils on the right occulted to show the field magnitude on vertical and horizontal planes.

a)

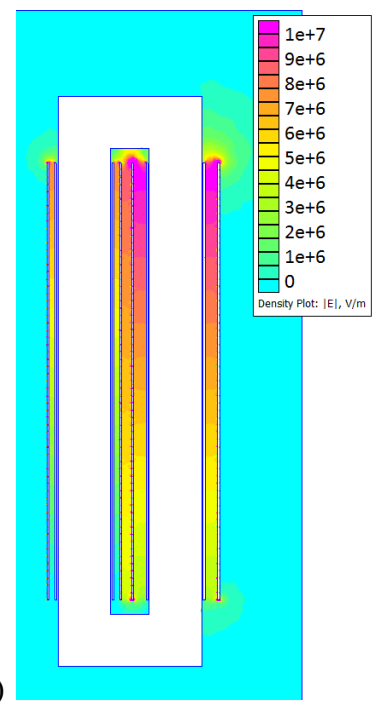

Fig. 13. Comparison between 2D a) and 3D b) FEA electrostatic field plots for the case $V_{1}=0$ and $V_{2}=150 \mathrm{kV}$. For 3D results, coils on the right occulted to show the field magnitude on vertical and horizontal planes.

is performed, one can notice that the results obtained by $2 \mathrm{D}$ and 3D FEA are in accordance. The differences between 2D and 3D FEA mainly occur in the windings corners where one can see that the magnetic field is more homogeneous than the electrostatic field. Consequently the error on the capacitances is bigger than the error on the leakage inductance. There is no significative difference between $2 \mathrm{D}$ and $3 \mathrm{D}$ approach for the magnetizing inductance estimation because only a negligible amount ot magnetizing energy is stored outside the core under no-load operation .

TABLE III

CHARACTERISTICS OF OPTIMAL CLIC TRANSFORMER CONFIGURATIONS.

\begin{tabular}{|l|c|c|c|}
\hline Characteristic & Constant space windings & Conical shape windings & Unit \\
\hline Weight & 264 & 255 & $\mathrm{Kg}$ \\
Dimensions & $0.407 \times 0.225 \times 0.678$ & $0.372 \times 0.240 \times 0.663$ & $\mathrm{~m}$ \\
\hline
\end{tabular}

\section{CONCLUSION}

An efficient optimal design methodology of monolithic high voltage pulse transformers based on the direct 2D FEA identification of the electrical equivalent circuit parameters has been presented. It can be used for a wide range of pulse transformer applications. This method has been successfully applied to the preliminary optimal design of the monolithic high voltage pulse transformer for the future CLIC modulators under study at CERN. The feasibility of such a transformer with tight specifications has been demonstrated. The predicted performances obtained with the direct 2D FEA optimization process have been compared and validated by 3D FEA simulation.

\section{ACKNOWLEDGMENT}

This work is supported by CERN, Switzerland and CRSNG, Canada.

\section{REFERENCES}

[1] D. Aguglia, C. A. Martins, M. C. Bastos, D. Nisbet, D. Siemaszko, E. Sklavounou and P. Viarouge, Klystron Modulator Technology Challenges for the Compact Linear Collider (CLIC), IEEE Pulsed Power conference (PPC), 2011, pp. 14131421.

[2] C.A. Martins, F. Bordry and G. Simonet, A solid state $100 \mathrm{kV}$ long pulse generator for Klystrons power supply," presented at the 13th European Conference on Power Electronics and Applications, EPE '09, Sept. 2009.

[3] M. Battaglia, A. De Roeck, J. Ellis, D. Schulte, Physics at the CLIC Multi-TeV Linear Collider - Report of the CLIC Physics Working Group, CERN report, ref. hep-ph/0412251; CERN-2004-005

[4] G. N. Glasoe, J. V. L, Pulse Generators, New York: Dover Publications, Inc., 1965

[5] Viarouge, P.; Aguglia, D.; Martins, C.A.; Cros, J., Modeling and dimensioning of High Voltage pulse transformers for klystron modulators, Electrical Machines (ICEM), 2012 XXth International Conference, On page(s): $2332-2338$

[6] Aguglia, D.; Viarouge, P.; Martins, C.A., Frequency domain non-linear identification method for high voltage pulse transformers, Electrical Machines (ICEM), 2012 XXth International Conference, On page(s): $1977-1983$ 